Rapid Reviews COVID-19

\title{
Review 1: "Obesity may hamper SARS-CoV-2 vaccine immunogenicity"
}

Jonathan Hare ${ }^{1}$

$\mathbf{1}_{\mathbf{A} \text { AVI }}$

Published on: May 07, 2021

License: Creative Commons Attribution 4.0 International License (CC-BY 4.0). 


\section{$\underline{\text { RR:C19 Evidence Scale rating by reviewer: }}$}

- Potentially informative. The main claims made are not strongly justified by the methods and data, but may yield some insight. The results and conclusions of the study may resemble those from the hypothetical ideal study, but there is substantial room for doubt. Decision-makers should consider this evidence only with a thorough understanding of its weaknesses, alongside other evidence and theory. Decisionmakers should not consider this actionable, unless the weaknesses are clearly understood and there is other theory and evidence to further support it.

******************************************

\section{Review:}

Claims are potentially informative by the data and methods used.

This manuscript addresses some fundamental themes that can contribute to the understanding of how the efficacy of COVID vaccines may vary across different physiological confounders. The work supports previous findings linking obesity with lower outcomes of vaccine immunogenicity in other diseases (influenza and hepatitis B).

This manuscript looks to evaluate binding antibody titer observed in a cohort of 248 Healthcare workers who have received two doses for the Pfizer/BioNTech BNT162b2 mRNA vaccine. Antibody tiers were determined at 7 days post second dose in agreement with previous published analysis. The data presented supports the hypotheses that obesity may contribute to lower vaccine immunogenicity, as determined by binding antibody titer. The authors hypothesize that there may be differences in the ability of the vaccine to offer protection at current dose regimen of $30 \mu \mathrm{g}$ and that different regimens for vaccination could be considered for individuals with elevated bmi. In this study there was not the data to show a worse clinical outcome for patients stratified by bmi, so whilst their hypothesis may be correct, larger mate analysis of clinical outcomes is required.

The work is accurately presented but the manuscript would require revisions for publication particularly around the structure and flow. Recommendations are below:

Do authors pay attention to ethics, diversity, and inclusion? Have the authors adequately discussed ethical concerns? When appropriate, have they been inclusive 
and taken into account equity, rights, and diversity?

There is no discussion of the ethics of engaging people on the basis of bmi and the practicalities of how this could be achieved. They do describe efforts for the use of consistent and harmonized definitions for obesity and more of the ethical considerations could be incorporated there.

This manuscript could be suitable for publication, but major revisions would be required.

- The language and flow of the manuscript needs to be improved. Some of the sentences are to staccato and don't relate to each other in a coherent manner. This is especially true in the introduction

- Some of the contents in the discussion, specifically around obesity and its impact on vaccine immunogenicity belongs in the introduction

- The authors should consider some of the ethical implications for their recommendations including practicalities. The authors could also consider approaching this discussion from the perspective of improving public health rather than bespoke vaccination approaches

- The statistical analysis should be included in the figures

- When data is discussed in the text but is not reflected in a figure please state that it is not shown. Alternatively, please consider drafting additional figures to support the analysis 